\title{
Rehabilitation am Lebensende - Neurophysiotherapie meets Palliative Care
}

Daniel Preiser und Rainer Simader

Physiotherapeuten, die mit Patienten mit neurologischen Erkrankungen arbeiten, finden sich immer wieder in Situationen, in denen auch das Sterben von Patienten möglich oder absehbar ist. Da Sterben nicht nur ein rein körperlicher Prozess ist, sondern auch mit vielen psychosozialen und auch spirituell-existenziellen Themen einhergeht, ein soziales Thema ist, An- und Zugehörige mit involviert sind und der Therapeut automatisch mit seiner eigenen Endlichkeit konfrontiert wird, ist Grundlagenwissen zu Palliative Care für die klinische Entscheidungsfindung relevant. In diesem Artikel erhalten Leser einen Einblick in die Palliative Care, und anhand von ausgewählten Situationen und Symptomen wird die Umsetzung in der neurologischen Rehabilitation skizziert.

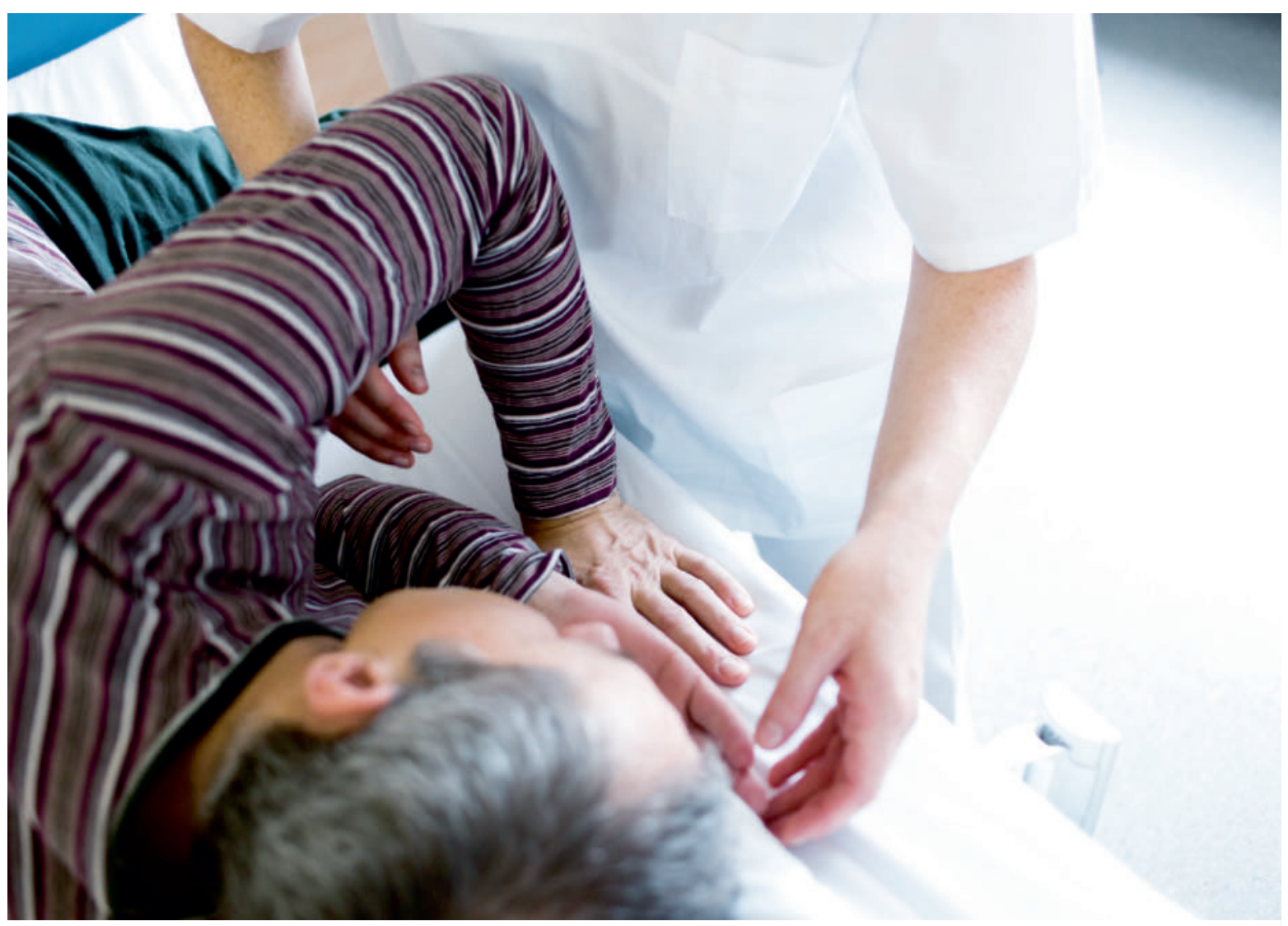

Grundlagenwissen zu Palliative Care ist für die klinische Entscheidungsfindung relevant.

(Quelle: A. Fischer/Thieme Gruppe; Symbolbild) 


\section{Palliative Care im Kontext neurologischer Rehabilitation}

Die WHO definiert Palliative Care folgendermaßen:

„Palliative Care ist ein Ansatz zur Verbesserung der Lebensqualität von PatientInnen und deren Familien, die mit Problemen konfrontiert sind, die mit einer lebensbedrohlichen Erkrankung einhergehen: durch Vorbeugen und Lindern von Leiden, durch frühzeitiges Erkennen, genaue Einschätzung und Behandlung von Schmerzen sowie anderen belastenden Beschwerden körperlicher, psychosozialer und spiritueller Art" [1].

Aus dieser Definition lassen sich mehrere Aspekte ableiten, die auch in der neurologischen Rehabilitation bekannt sind: Es geht um die Verbesserung der Lebensqualität, sehr oft um das Miteinbeziehen des sozialen Systems und schließlich auch um die Berücksichtigung von vielen Dimensionen einer Erkrankung - und nicht nur rein um die körperliche Thematik. Genauso wie die Palliative Care bedient sich auch die Neurologie oftmals eines interdisziplinären Teams.

Diese Definition besagt auch, dass es generell um lebensbedrohliche Erkrankungen geht, oder, wie Radbruch et al. [2] feststellen, um Patienten mit fortgeschrittenen und progredienten Erkrankungen, die eine begrenzte Lebenserwartung haben. Fälschlicherweise wird Palliative Care noch immer hauptsächlich mit Tumorerkrankungen assoziiert. Kardiorespiratorische Erkrankungen, Multimorbidität, chronische Krankheitsverläufe durch die zunehmende Lebenserwartung benötigen ebenso Palliativversorgung wie viele Patienten mit progredienten neurodegenerativen Erkrankungen (Morbus Parkinson, ALS, Multiple Sklerose, Demenz) oder Menschen mit schweren erworbenen Hirnschäden (Unresponsive Wakefulness Syndrome, Minimally Conscious State).

Die WHO-Definition geht auch auf den zeitlichen Aspekt des Einsatzes von Palliative Care ein. Es ist nicht möglich und wäre auch nicht sinnvoll, in absoluten Zahlen zu beschreiben, wie lange vor dem Tod Palliative Care beginnen soll. Erstens könnte eine genaue Zeitangabe immer nur retrospektiv, also wenn der Zeitpunkt des Todes bekannt ist, gestellt werden, und zum anderen sind Palliative Care und Kurativmedizin keine „Kontrahenten“, sondern im Prozess von schweren Erkrankungen Partner, bei denen einmal der eine Ansatz überwiegt, dann der andere [3], bis in der Regel am Ende des Lebens die Palliative Care im Vordergrund steht. Eine palliative Begleitung kann über Jahre dauern. Genau deswegen beschreibt die WHO auch den wichtigen Ansatz der Vorbeugung.

Das Einbeziehen von Palliativexperten in einer früheren Phase von Erkrankungen, die aber dennoch mit hoher Wahrscheinlichkeit zum Tod führen werden, ist in der
Therapieplanung wichtig. Gerade weil Menschen mit neurodegenerativen Erkrankungen in späteren Phasen des Lebens oft nicht mehr zu ihrer eigenen Situation und zum bevorstehenden Lebensende kommunizieren können, kommt diesem frühen Einsatz eine besondere Bedeutung zu [4].

\section{Rehabilitation am Lebensende?}

Betrachtet man den Ursprung des Wortes Rehabilitation, also „rehabilitare“, so bedeutet dies „wiederbefähigen“. Viele Menschen am Lebensende verlieren kontinuierlich oder auch plötzlich Fähigkeiten. Dazu kommt, dass Menschen an ihrem Lebendende oft nicht ausreichend gefördert und gefordert werden. Dies kann verursacht werden durch die Befürchtung, Situationen zu verschlechtern, durch vermindertes Zutrauen und durch die eigene Haltung und Vorstellungen, wie Sterben zu sein hat. Betrachtet man die ICF, die genauso wie in der Neurologie auch in der palliativen Physiotherapie ein Fundament des Clinical Reasonings ist [5], so sind entscheidende rehabilitative Zielsetzungen die (Wieder-)Befähigung zur sozialen Teilhabe, die bestmögliche Aktivität und die optimale Symptomkontrolle.

\section{Hilfreiche Haltung und Zielsetzung in der Palliative Care}

Cicely Saunders, die Begründerin des modernen Hospizwesens, hat folgenden hilfreichen Satz geprägt:
„Die Arbeit des professionellen Teams ... ist, dass wir alles tun, dem Patienten Leben zu ermöglichen, bevor er stirbt - unter Berücksichtigung seines maximalen Potenzials, der Grenzen seiner körperlichen und psy- chischen Möglichkeiten und der Selbstkontrolle und Unabhängigkeit, wann immer auch möglich“ [6].

Das, was beinahe wie ein Stellenprofil für einen Physiooder Ergotherapeuten klingt, beschreibt aber die eigentliche Aufgabe, die wir haben, wenn wir schwer kranke und sterbende Menschen begleiten - unabhängig von deren Grunderkrankung und dem Stadium einer Erkrankung. Dieses Zitat soll auch unseren Blickwinkel auf Menschen mit schweren Erkrankungen justieren. Saunders schreibt von Potenzialen, von Selbstkontrolle, von Möglichkeiten und letztlich von Unabhängigkeit - soweit möglich. Deshalb ist es die Aufgabe in der neurologisch-palliativen Therapie, nach diesen Potenzialen zu suchen oder, wie Gerhard [3] beschreibt, eine „suchende Haltung“ einzunehmen. Peter Nieland, der Botschafter der Physiotherapie in der Palliative Care in Deutschland, drückte es so aus: „Es gehört auch zur Würde des Menschen, ein Glas selbstständig zum Mund führen zu können“ [7].

Mit diesem Auftrag von Cicely Saunders geht auch die Frage einher, was wir schwer kranken und sterbenden 
Menschen zutrauen und welchen Blick wir auf Sterbende werfen. Sehen wir primär den leidenden und schwer kranken Menschen oder sehen wir auch den mutigen Helden, der auf seine letzte Reise geht? Je nachdem, welchen Blick wir haben und was wir einem leidenden Menschen oder aber einem Helden zutrauen, ergeben sich mitunter auch unterschiedliche Therapieansätze.

\section{Lebensqualität und Lebensängste am Lebensende}

Menschen am Lebensende wird von Außenstehenden oft eine schlechte Lebensqualität attestiert. Gerade im neurologischen Setting, in dem Patienten des Öfteren kognitive und kommunikative Einschränkungen haben, sind die begleitenden Berufsgruppen häufig von Aussagen von nahen Angehörigen abhängig. Allerdings ist hier zu beachten, dass Angehörige die Lebensqualität oft schlechter einschätzen als die Patienten selbst [8]. Gerhard [3] beschreibt dieselbe Diskrepanz zwischen Ärzten/Pflegekräften und Patienten mit Krebserkrankungen - auch bei an ALS (Amyotrophe Lateralsklerose) erkrankten Patienten ist die subjektive Lebensqualität in vielen Fällen sehr gut [3]. Dennoch ist Lebensqualität ein sehr individueller Aspekt und kann nur mit den Patienten persönlich evaluiert werden. Wenn dies nicht (mehr) möglich ist, so müssen der mutmaßliche Wille und somit die mutmaßliche Lebensqualität eruiert werden.

Die häufigsten Ängste und Sorgen von Menschen am Lebensende sind u. a. die Angst vor unkontrollierten Symptomen, die Angst, anderen Menschen zur Last zu fallen, die Angst, die Selbstständigkeit zu verlieren, oder etwa - bei älteren Menschen - zu stürzen. Umgekehrt gehören z. B. körperliche Funktion und Unabhängigkeit zu sehr wichtigen Parametern der Lebensqualität bei Palliativpatienten [9-11]. Auch wenn Einflussfaktoren auf die Lebensqualität am Lebensende generell noch besser erforscht werden müssen, so spielen körperliche Funktion, Aktivität, Selbstständigkeit und Partizipation bis zuletzt eine wesentliche Rolle.

\section{Bandbreite der Therapie}

Die Bandbreite der Rehabilitation in der Palliative Care ist sehr vielfältig [12]:

- vorsichtige Übungen mit Hilfe im Bett

- im Stuhl sitzen

- Transfer vom Sitzen zum Stehen

- Gehen mit Unterstützung

- selbstständiges Gehen

- Treppensteigen

- leichtes Zirkeltraining

Geht man in Hospize in England, sollte man nicht überrascht sein, wenn man dort Fitnessstudios (sog. „Rehabilitation Gyms“) für Patienten findet.
Motorische Leistungen können bis zum Lebensende verbessert werden, und diese sind wichtige Bausteine für die Aktivität und Teilhabe. Deswegen ist es wesentlich, dass Potenziale im gesamten Krankheitsverlauf - bis zuletzt - gefördert werden.

\section{Die Plastizität bis zuletzt positiv beeinflussen}

Unter Neuroplastizität versteht man die (lebenslange) Fähigkeit des Gehirns, seine Struktur und Organisation kontinuierlich an neue Umgebungsbedingungen anzupassen [13]. Diese Anpassungsfähigkeit besteht auch bei Erkrankungen oder nach Verletzungen des zentralen Nervensystems und kann durch körperliche Aktivität positiv beeinflusst werden [14]. Abhängig von der Art der Einflüsse können diese für den Patienten nützlich sein oder eben nicht. Dies macht den Umgang insbesondere mit schwer betroffenen Patienten so entscheidend.

Die Herausforderung für ein Therapieteam ist es, die Reaktionen des Patienten zu beobachten, interpretieren zu lernen und seine Ressourcen zu erkennen. Ein darauf aufbauender Zugang zum Patienten schafft die Möglichkeit, (bis zuletzt) sinnvolle und effiziente sensomotorische Funktionen zu fördern, Sekundärschäden zu verhindern und Alltagspartizipation zu ermöglichen.

\section{Der Sinn von Bewegungen und der Therapie}

Oft sind Maßnahmen zur Prophylaxe von Sekundärschädigungen wie „das Durchbewegen“ bei bestehender Immobilität von Patienten Inhalt der Therapie. Geschieht dies in Form von Positionswechseln, in einem sinnvollen Alltagskontext (z. B. Einnehmen einer bequemen Ruheposition oder Aufsetzen zur Oberkörperpflege), unter Einsatz von stabilisierendem und orientierendem Lagerungsmaterial, einer umfassenden Anregung des gesamten Sensoriums inklusive der propriozeptiven vestibulären und optischen Aktivierung, vermittelt es dem Patienten Orientierung am eigenen Körper, verbessert den Gasaustausch und schafft Sicherheit.

Im Gegensatz dazu werden beim passiven Durchbewegen in Rückenlage die Extremitäten des Patienten gegen einen stillgelegten Rumpf bewegt. Schulter- und Beckengürtel können gegen die Schwerkraft nicht (genügend) mitbewegen, der sensorische Input ist somit auf die Extremitäten begrenzt. Das Bewegungstempo ist häufig rascher als beim ganzheitlichen Fazilitieren. Aspekte wie Pneumonieprophylaxe, vegetative Anregung und Kreislaufaktivierung werden nicht ausreichend berücksichtigt. Zusätzlich wird die Umwelt aus der Rückenlage in einem ungünstigen Blickwinkel gesehen, die abstrakten Bewegungen sind nicht funktionell und können vom Patienten nicht mit sinn- 
vollen, alltagsrelevanten Bewegungen assoziiert werden; somit bleiben sie bedeutungslos.

\section{Beispiel: Mobilisation in den Rollstuhl}

Die Mobilisation in den Rollstuhl hat eine hohe Relevanz für Patienten. Sie ermöglicht es ihnen, das Bett zu verlassen, und schafft die Voraussetzung für räumliche und örtliche Mobilität. Kommt es durch längere Immobilität oder neurologische Schädigung zu einer Störung des Körperschemas [15], können Patienten Probleme mit der posturalen Orientierung haben [16]. Bei der Vertikalisierung in den Sitz kann der Verlust von Sicherheit durch die Orientierungsstörung Angst verursachen und dadurch die Motivation und die Kooperation des Patienten erheblich bremsen. Als wichtige Afferenzen für das Körperschema können Füße, Hände, unterer Rumpf, HWS und das optische System stimuliert werden (siehe Positionswechsel). Stabilisierendes Lagerungsmaterial und der Körper des Therapeuten können räumliche Orientierung schaffen, um so eine im Tempo an den Patienten angepasste Mobilisation in den Sitz zu gestalten bzw. einen Transfer in den Rollstuhl zu erarbeiten.

Dennoch ist das Prinzip der Autonomie in der Palliative Care ein zentrales Element. Ablehnung von Mobilisierung kann z. B. Ausdruck eines Autonomiewunsches, eines dahinterliegenden Problems oder Bedürfnisses sein. Es geht darum, solche Aussagen mit dem Patienten und/oder im Team zu besprechen, um herauszufinden, welche Gründe zur Abwehr führen. So kann die Ablehnung der Mobilisation z. B. daran liegen, dass der Patient Angst vor Schmerzen hat, dass er bei der letzten Mobilisation unangenehme Erfahrungen gemacht hat oder aber, weil er einfach nicht will. Dieser „Blick hinter Aussagen oder Verhalten“ ist essenziell für die Lebensqualität.

\section{Beispiel Schmerz}

Schmerz ist ein häufiges Symptom bei schweren Erkrankungen und am Lebensende. Bei Schmerzen sollte beim Bewegen der schmerzfreie Bewegungsradius angestrebt und ausgenutzt werden. Unter Beobachtung seiner Reaktionen sollte jede sinnvolle Aktivität des Patienten genützt und ausgebaut werden. Eine maximal stabile, sichere Endposition vermeidet unnötige Kraftaufwendung durch Haltearbeit, reduziert Schmerzen und ermöglicht Partizipation.

Die Fähigkeit, Schmerzen zu verbalisieren, kann in vorgeschrittenen Stadien neurodegenerativer Erkrankungen verloren gehen. Patienten hängen dann von einer externen Schmerzbeurteilung durch geschultes medizinisches Fachpersonal ab, die zu diesem Zweck existierende gut validierte Bewertungsinstrumente nutzen sollten $[4,18]$. Und dennoch kann im Kontext des Lebensendes beobachtet werden, dass trotz höchster Achtsamkeit und entspre- chender Medikation Schmerzen bleiben oder sich anders als erwartet verhalten.

Total-Pain-Konzept In der Palliative Care ist das TotalPain-Konzept, ebenfalls von Cicely Saunders entwickelt, eine bedeutsame Betrachtungsweise von Schmerzen (und auch anderen Symptomen) [19-20]. Viele Therapeuten kennen schmerzgeplagte Patienten, die aber in bestimmten Situationen, z. B. wenn die Enkelkinder im Krankenbett hüpfen, wie ausgewechselt erscheinen. Der Schmerz scheint dann keine Bedeutung zu haben. Das Total-PainKonzept beschreibt, dass es neben den rein körperlichen Faktoren viele Einflussfaktoren gibt, die negativ, aber auch positiv auf den Schmerz wirken. Dies sind emotionale, soziale und auch spirituell-existenzielle Aspekte, welche die Wahrnehmung und auch die Bedeutung von Schmerz verstärken oder erleichtern können. Manchmal ist dann auch der Seelsorger der beste Schmerztherapeut - oder ein Telefonat mit einem weit entfernten Freund oder das vermisste Haustier, das nun am Bett liegt, tragen zur Verbesserung der Schmerzsituation bei. Gerade im Kontext des Lebensendes muss dieser Ganzheitlichkeit eine hohe Aufmerksamkeit entgegengebracht werden, und oft sind Symptome multifaktoriell beeinflusst und beeinflussbar.

\section{Beispiel Fatigue}

Fatigue ist eine ausgeprägte Müdigkeit und Erschöpfung, die Krankheitswert hat und nichts mit einer vorangegangenen Aktivität zu tun hat [19]. Es ist eines der häufigsten Symptome am Lebensende und auch eines, das am häufigsten nicht ausreichend diagnostiziert und behandelt wird. Genauso leiden Patienten mit neurologischen Erkrankungen oft unter diesem belastenden Symptom. Fatigue hat sowohl körperliche, emotionale als auch kognitive Auswirkungen und wird durch ebensolche Faktoren (z. B. Bewegungsmangel, Reizarmut, Langeweile oder etwa Depression) verstärkt.

Anders als bei „normaler Müdigkeit“ helfen demnach Ausruhen und Schlafen nicht, sondern es sind hauptsächlich aktivitätsfördernde Ansätze bis hin zu Training, gepaart mit verhaltenstherapeutischen Ansätzen wie Tagesplanung, Strategien zur Energiebereitstellung und auch Edukation für die Behandlung relevant. Da es kaum medikamentöse Behandlungsansätze gibt, beschreibt die Europäische Palliativgesellschaft (EAPC), dass gerade Physiotherapie als nonpharmakologische Intervention hier eine besondere Rolle spielt [21].

\section{Edukation bietet Sicherheit}

Dem „Empowerment“, also der Selbstbefähigung von Patienten, der bestmöglichen Selbstkontrolle und Autonomie und dem Bewahren der Würde wird in der Palliative Care ein hoher Wert beigemessen. Edukation ist dazu ein wesentliches Mittel. Wenn Patienten verstehen, warum 
und wie ein Vorgehen sein wird, so trägt dies zur besseren Akzeptanz und auch Wirkung der Maßnahmen bei und unterstützt die eben genannten Aspekte der Selbstkontrolle. In der Neurologie sehen sich Therapeuten oft Patienten gegenüber, die nicht ausreichend in der Lage sind, kognitiven edukativen Ansätzen zu folgen. In der palliativen neurologischen Therapie ist es wichtig, die Möglichkeiten der Edukation weiter zu fassen. Auch körperliche und emotionale Botschaften bieten dem Patienten diese Sicherheit.

Bei verbalen Ansätzen sind zum einen die professionelle Beratung, Anleitung und Information von An- und Zugehörigen wichtig. In der Kommunikation mit dem Patienten sind einfache, geschlossene Fragen und ein regelmäßiges Ansprechen mit einem möglichst natürlichen Tonfall, das Ankündigen von Maßnahmen, Lob und kontinuierliche Rückmeldung hilfreich. Dabei sind emotionale Botschaften oftmals besser verständlich als rein kognitive.

Neben den verbalen bieten auch körperorientierte Ansätze wichtige Informationen für den Patienten. Dazu gehören:

- Rituale schaffen, die Sicherheit geben

- Bieten eines sicheren Raumes

- Durchführen von globalen Bewegungsübergängen und somit Abrufen von altbekannten Programmen mit Alltagsrelevanz

- räumliche Orientierung geben

„Speed kills“ Dies ist nicht nur im Straßenverkehr eine Tatsache, sondern auch, im übertragenen Sinne, bei jeglichem Umgang mit schwer kranken und sterbenden Menschen - in der Kommunikation genauso wie in der Durchführung der Therapie. Dazu gehört eine hohe Achtsamkeit, auch gegenüber der Tagesform der Patienten. Therapieprozesse in der palliativen Rehabilitation fluktuieren tageweise und kontinuierlich über die gesamte Begleitung hinweg. Das Ziel kann Besserung oder Stabilisierung einer Situation sein, bis hin zur Begleitung durch die sicher auftretenden Phasen der Verschlechterung. Die Funktionsfähigkeit nimmt mit zunehmendem Krankheitsverlauf ab, und der Therapeut darf dennoch den ressourcenorientierten, achtsamen und rehabilitativen Ansatz nicht aus den Augen verlieren. - Abb. 1 veranschaulicht unterschiedliche Verläufe von Funktionsfähigkeit.

\section{Fazit}

Wenn Aktivitäten und Bewegungen dem Patienten sinnvoll erscheinen, wenn er, je nach Möglichkeiten, versteht, warum diese durchgeführt werden, und der Therapeut durch seine spezifische Arbeit die Entwicklung des Patienten und seines Umfelds bestmöglich beeinflusst und dabei achtsam auf seine Reaktionen eingeht, so wird diese Therapie zu einer Verbesserung der Lebensqualität auch am Lebensende des Patienten beitragen.

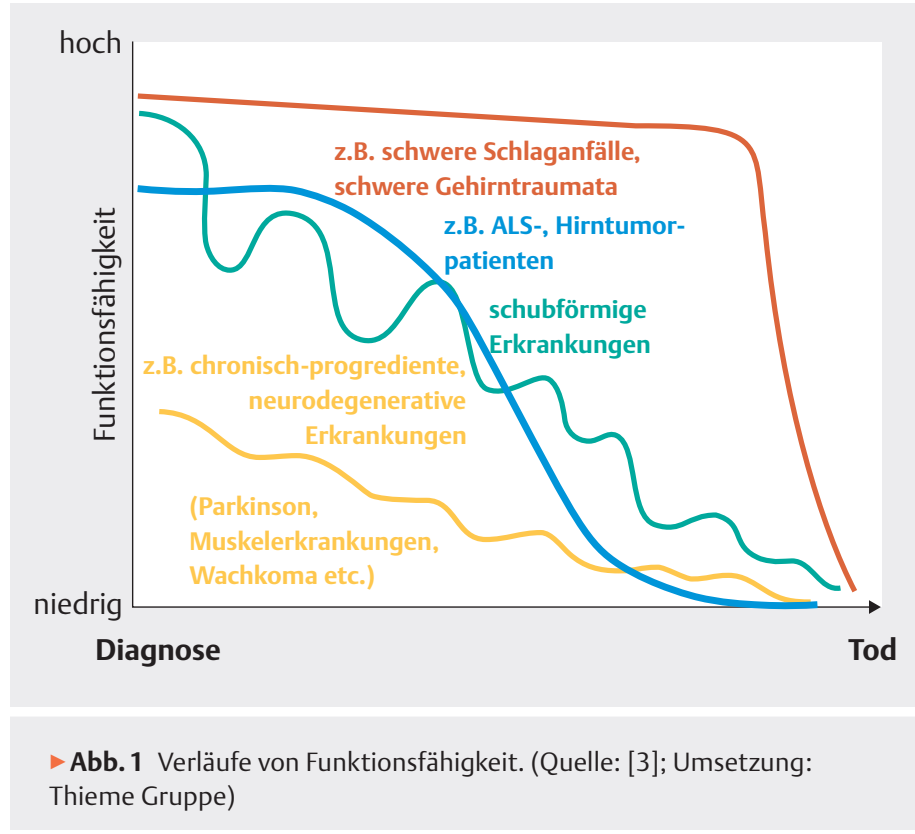

Autorinnen/Autoren

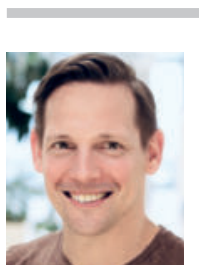

\section{Daniel Preiser}

Ist Physiotherapeut und hat einen Master in Physiotherapie. Seine Arbeitsschwerpunkte bilden die therapeutische Leitung der ambulanten neurologischen Rehabilitation, Betreuung der Wachkomastation und der Multiple-Sklerose-Station im Haus der Barmherzigkeit in Wien sowie eine freiberufliche Tätigkeit im Behandlungsfeld Neurologie.

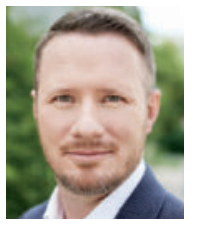

\section{Rainer Simader}

Ist Physiotherapeut und Autor und hat die Fachgruppe Physiotherapie in der Palliative Care bei PhysioAustria gegründet. Er leitet das Ressort Bildung bei Hospiz Österreich, dem Dachverband aller österreichischen Hospizund Palliativeinrichtungen und ist Mitglied des Leitungsteams des Universitätslehrganges Palliative Care in Salzburg.

\section{Korrespondenzadressen}

\section{Daniel Preiser}

E-Mail: daniel.preiser@hb.at www.neurophysiowien.com

\section{Rainer Simader}

E-Mail: simaderr@gmail.com

www.rainer-simader.com 
Literatur

[1] Dachverband Hospiz Österreich. Die Idee von Hospiz und Palliative Care. Im Internet: www.hospiz.at/hospiz-palliativecare/die-idee-von-hospiz-und-palliative-care/; Stand: 20.7.2020

[2] Radbruch L et al. Definitionen, Entwicklung und Ziele. In: Aulbert E, Nauck F, Radbruch L, Hrsg. Lehrbuch Palliativmedizin. 2. Aufl. Stuttgart: Schattauer; 2007

[3] Gerhard C. Neuro-Palliative Care. Bern: Huber; 2011

[4] Lorenzl S et al. Handbook of Clinical Neurology, Vol. 118 (3 rd series): Ethical and Legal Issues in Neurology. Elsevier; 2013

[5] Dahlin Y. Der richtige Zeitpunkt: Gibt es ein „zu früh“ oder ein „zu spät“ für die Physiotherapie? In: Nieland P, Simader R., Taylor J, Hrsg. Was wir noch tun können: Rehabilitation am Lebensende. München: Elsevier; 2013

[6] Saunders C. Foreword. In: Doyle D et al., eds. Oxford Textbook of Palliative Medicine. New York: Oxford University Press; 1998

[7] Simader R. Physiotherapie in der Palliativversorgung. In Höppner H, Kühnast K, Winkelmann C. Potenziale der Physiotherapie erkennen und nutzen. Heidelberg: medhochzwei; 2020

[8] Hack T et al. Assessing symptoms, concerns, and quality of life in noncancer patients at end of life: How concordant are patients and family proxy members? Journal of Pain and Symptom Management 2018; 56: 760-766

[9] Nieland P, Simader R, Taylor J. Was wir noch tun können: Rehabilitation am Lebensende. München: Elsevier; 2013

[10] Oldervoll et al. The effect of a physical exercise program in palliative care. J Pain Symptom Manage 2006; 31: 421-430

[11] Ruppe et al. Österreichische interdisziplinäre Hochaltrigenstudie. Individuelle und soziale Potenziale für ein "gutes Leben“ im hohen Alter. Österreichische Plattform für Interdisziplinäre Alternsfragen (ÖPIA); 2018

[12] Taylor J. Von der Symptomkontrolle zur Rehabilitation. In: Nieland P, Simader R, Taylor J. Was wir noch tun können: Rehabilitation am Lebensende. München: Elsevier; 2013
[13] Nelles G et al. Rehabilitation von sensomotorischen Störungen, S2k-Leitlinie, 2018. In: Deutsche Gesellschaft für Neurologie, Hrsg. Leitlinien für Diagnostik und Therapie in der Neurologie. Im Internet: www.dgn.org/leitlinien; Stand: 20.08.2020

[14] Kempermann G, Fabel K, Ehninger D et al. Why and how physical activity promotes experience-induced brain plasticity. Front Neurosci 2010; 4: 189. doi:10.3389/fnins.2010.00189

[15] Haggard P, Wolpert D. Disorder of body scheme. In: Freund HJ, Jeannerod M, Hallett M, Leiguarda R, eds. Higher-Order Motor Disorders. Oxford: Oxford University Press; 2005

[16] Horak FB. Postural orientation and equilibrium: What do we need to know about neural control of balance to prevent falls? Age and Ageing 2006; 35: ii7-ii11. doi:10.1093/ ageing/afl077

[17] Lorenzl S et al. Handbook of Clinical Neurology, Vol. 118 (3 rd series): Ethical and Legal Issues in Neurology. Elsevier; 2013

[18] Laekeman M. Pain assessment in impaired cognition scale (PAIC 15). physiopraxis 2020; 18(06): 52-53

[19] Coym A, Hlawatsch C. In: Oechsle K, Scherg A, Hrsg. FAQ Palliativmedizin. München: Elsevier; 2019

[20] Saunders C. Care of the dying. Current Medical Abstracts for Practitioners 1963; 3: 77-82

[21] Radbruch $L$ et al. Fatigue in palliative care patients: An EAPC approach. Palliative Medicine 2008; 22: 13-32

Bibliografie

DOI https://doi.org/10.1055/a-1284-8903

neuroreha 2020; 12: 163-168

(c) 2020. Thieme. All rights reserved.

Georg Thieme Verlag KG, Rüdigerstraße 14

70469 Stuttgart, Germany

ISSN 1611-6496 